\title{
Postoperative atrial fibrillation and total dietary antioxidant capacity in patients undergoing cardiac surgery: The Polyphemus Observational Study
}

\author{
Simona Costanzo, MSc, PhD, ${ }^{\mathrm{a}}$ Amalia De Curtis, BSc, ${ }^{\mathrm{a}}$ Veronica di Niro, BN, ${ }^{\mathrm{b}}$ Marco Olivieri, IT, \\ Mariarosaria Morena, BN, ${ }^{\mathrm{d}}$ Carlo Maria De Filippo, MD, ${ }^{\mathrm{d}}$ Eugenio Caradonna, MD, ${ }^{\mathrm{d}}$ Vittorio Krogh, MD, \\ Mauro Serafini, MSc, ${ }^{f}$ Nicoletta Pellegrini, MSc, PhD ${ }^{g}$ Maria Benedetta Donati, $\mathrm{MD}, \mathrm{PhD}$, \\ Giovanni de Gaetano, $\mathrm{MD}, \mathrm{PhD},{ }^{\mathrm{a}}$ and Licia Iacoviello, $\mathrm{MD}, \mathrm{PhD},{ }^{\mathrm{a}}$ on behalf of the Polyphemus \\ Observational Study Investigators
}

Objective: Postoperative atrial fibrillation is a major cause of morbidity and mortality for stroke after cardiac surgery. Both systemic inflammation and oxidative stress play a role in the initiation of postoperative atrial fibrillation after cardiac surgery. The possible association between long-term intake of antioxidant-rich foods and postoperative atrial fibrillation incidence was examined in patients undergoing cardiac surgery.

Methods: A total of 217 consecutive patients ( $74 \%$ were men; median age, 68.4 years) undergoing cardiac surgery, mainly coronary artery bypass grafting and valve replacement or repair, were recruited from January 2010 to September 2012. Total antioxidant capacity was measured in foods by the Trolox equivalent antioxidant capacity assay. The European Prospective Investigation into Cancer and Nutrition Food Frequency Questionnaire was used for dietary total antioxidant capacity assessment. The association among tertiles of dietary total antioxidant capacity and postoperative atrial fibrillation incidence was assessed using multivariable logistic analysis.

Results: The overall incidence of total arrhythmias and postoperative atrial fibrillation was $42.4 \%$ and $38.2 \%$, respectively. In multivariable analysis, after adjustment for age, gender, use of hypoglycemic drugs, physical activity, education, previous diagnosis of atrial fibrillation, and total energy intake, patients in the highest tertile of dietary total antioxidant capacity had a lower risk of postoperative atrial fibrillation than patients in the 2 lowest tertiles (odds ratio, $0.46 ; 95 \%$ confidence interval, $0.22-0.95 ; P=.048)$. A restricted cubic spline transformation confirmed the nonlinear relationship between total antioxidant capacity (in continuous scale) and postoperative atrial fibrillation $(P=.023)$. When considering only coronary artery bypass grafting, valve replacement/repair, and combined surgeries, the protective effect on postoperative atrial fibrillation of a diet rich in antioxidants was confirmed.

Conclusions: Long-term consumption of antioxidant-rich foods is associated with a reduced incidence of postoperative atrial fibrillation in patients undergoing cardiac surgery. (J Thorac Cardiovasc Surg 2015;149:1175-82)

\footnotetext{
From the Department of Epidemiology and Prevention, ${ }^{\mathrm{a}}$ IRCCS Istituto Neurologico Mediterraneo Neuromed, Pozzilli (IS), Italy; Nuovo Ospedale Civile di Sassuolo, ${ }^{\text {b }}$ Sassuolo, Italy; EPICOMED Research, ${ }^{\mathrm{c}}$ srl, Campobasso, Italy; Department of Cardiovascular Disease, ${ }^{\mathrm{d}}$ Fondazione di Ricerca e Cura "Giovanni Paolo II," Campobasso, Italy; Epidemiology and Prevention Unit, ${ }^{e}$ Fondazione IRCCS Istituto Nazionale dei Tumori, Milan, Italy; Functional Food and Metabolic Stress Prevention Laboratory, ${ }^{\mathrm{f}}$ Agricultural Research Center, CRA-NUT, Rome, Italy; and Department of Food Science, ${ }^{\mathrm{g}}$ University of Parma, Parma, Italy.

This study was supported by the Italian Ministry of Health, Young Researchers Grant Number 2008-1146478, The European Foundation for Alcohol Research (ERAB) Grant EA082, The International Organisation of Vine and Wine (OIV) Grant 2011 to S.C., and the Project "Malattie cardiovascolari: ruoli di fattori genetici, acquisiti, nuovi approcci terapeutici e condizioni organizzative ottimali per la produzione delle conoscenze" (D. MIUR n. 328 del 01/07/2010). These funding sources had no involvement in the study design; the collection, analysis, and interpretation of data; the writing of the article; or the authors' decision to submit the article for publication. Disclosures: Authors have nothing to disclose with regard to commercial support.

The Polyphemus study investigators are listed in the Appendix E1.

Received for publication July 2, 2014; revisions received Oct 15, 2014; accepted for publication Nov 7, 2014; available ahead of print Dec 19, 2014.

Address for reprints: Simona Costanzo, MSc, PhD, Department of Epidemiology and Prevention, IRCCS Istituto Neurologico Mediterraneo Neuromed, Via Atinense 18, 86077 Pozzilli, IS, Italy (E-mail: simona.costanzo@neuromed.it). $0022-5223 / \$ 36.00$

Copyright (c) 2015 by The American Association for Thoracic Surgery http://dx.doi.org/10.1016/j.jtcvs.2014.11.035
}

See related commentary on pages $1182-4$.

丹 Supplemental material is available online.

Atrial fibrillation (AF) frequently occurs after an open heart surgery and is associated with a high risk of cardiovascular and cerebrovascular death ${ }^{1}$ and postoperative stroke, ${ }^{2}$ with consequent prolonged hospitalization and increased total hospital costs. Several epidemiologic studies show an AF incidence ranging from $20 \%$ to $50 \%$, and this large variation depends on the different characteristics of patient populations, complexity of the surgical procedure, and method of arrhythmia surveillance. ${ }^{3,4}$

The pathophysiology of AF after an open heart surgery is multifactorial and still not well known, so its management is difficult. Several strategies to reduce its occurrence 


$$
\begin{aligned}
& \text { Abbreviations and Acronyms } \\
& \text { AF }=\text { atrial fibrillation } \\
& \text { CABG }=\text { coronary artery bypass grafting } \\
& \text { CI }=\text { confidence interval } \\
& \text { FRAP }=\text { ferric reducing antioxidant power } \\
& \text { ICU }=\text { intensive care unit } \\
& \text { OR }=\text { odds ratio } \\
& \text { POAF }=\text { postoperative atrial fibrillation } \\
& \text { TAC }=\text { total antioxidant capacity } \\
& \text { TEAC }=\text { Trolox equivalent antioxidant capacity } \\
& \text { TRAP }=\text { radical-trapping antioxidant parameter } \\
& \text { VR }=\text { valve replacement or repair }
\end{aligned}
$$

have been investigated. Numerous risk factors associated with the development of postoperative atrial fibrillation (POAF) have been reported, such as advanced age, hypertension, diabetes, obesity and metabolic syndrome, intraoperative factors (surgical injury, atrial ischemia, pulmonary vein vent, venous cannula), and postoperative factors (volume overload, increased afterload, and hypotension). ${ }^{4,5}$ The main common pathways of all these factors are inflammation and oxidative stress. ${ }^{4,6,7}$

Pharmacologic (eg, beta-blockers and amiodarone) and nonpharmacologic (eg, atrial pacing) treatments have been used to prevent POAF, but all therapeutic options have shown variable efficacy. Currently, in addition to the traditional antiarrhythmic therapy, new therapies (nonantiarrhythmic drugs and nutraceuticals) have received increasing attention for preventing POAF. ${ }^{8,9}$

We reasoned that the long-term consumption of a diet rich in antioxidant foods before cardiac surgery could prevent oxidative stress and inflammation by scavenging reactive oxygen and reactive nitrogen species. The evaluation of the total antioxidant capacity (TAC) of the diet allows the assessment of the antioxidant activity of food components and their synergic interactions, providing comprehensive information about dietary antioxidant intake. ${ }^{10}$

Several studies have shown that dietary TAC is inversely related to C-reactive protein, ${ }^{10}$ metabolic syndrome, ${ }^{11}$ total mortality, ${ }^{12}$ and stroke in general population, ${ }^{13}$ and to myocardial infarction ${ }^{14}$ in women. In view of the paucity of data on dietary TAC in relation to arrhythmias, the main objective of this study was to evaluate and characterize the possible negative association between dietary TAC and POAF incidence in patients undergoing open heart surgery.

\section{MATERIALS AND METHODS \\ Recruitment Strategy}

Consecutive patients undergoing cardiac surgery were recruited the Department of Cardiovascular Disease of the Fondazione di Ricerca e Cura "Giovanni Paolo II," Catholic University, Campobasso, Italy, from January 2010 to September 2012.
The inclusion criteria were age 18 years or more and being scheduled for the following cardiac surgeries: coronary artery bypass grafting (CABG), valve replacement or repair (VR), aortic aneurysm, or their combinations. Use of antiarrhythmic drugs (chronic or prophylactic therapy) and a history of arrhythmia, in particular AF, were not exclusion criteria. All patients signed an informed consent to participate in the study, which was approved by the Ethical Committee of the Catholic University of the Sacred Heart, Rome, Italy.

Trained interviewers administered a structured questionnaire to collect personal and clinical information, including socioeconomic status, physical activity, medical history, drug use, risk factors for cardiovascular disease, and dietary habits in the last year. Additional clinical information, such as electrocardiography, echocardiography, coronarography, drug therapy during hospitalization, perioperative course, and intra-hospital complications, were collected by checking patients' medical records.

\section{Total Dietary Antioxidant Capacity Assessment}

The European Prospective Investigation into Cancer and Nutrition Food Frequency Questionnaire adapted to the Italian population was used to determine usual nutritional intakes in the past year. The Nutritional Analysis of Food Frequency Questionnaire software (National Cancer Institute, Milan, Italy) was used to transform information about food composition into daily intake of food items, energy, macronutrients, micronutrients, and TAC. ${ }^{15}$ Nutrient data for specific foods were obtained from the food composition database for epidemiologic studies in Italy integrated with the TAC values of a number of foods representative of the average Italian diet, such as fruits, vegetables, oils, beverages, spices, dried fruits, sweets, cereals, pulses, and nuts. $^{16-18}$

The TAC database has been widely validated in the Italian popula$\operatorname{tion}^{10,13}$ and in other Mediterranean populations (Spain and Greece). ${ }^{12,19}$ TAC was measured in foods by 3 different assays: the Trolox equivalent antioxidant capacity (TEAC) assay measuring the antioxidant's ability to reduce a radical reaction in both lipophilic and hydrophilic conditions, the radical-trapping antioxidant parameter (TRAP), and ferric reducing antioxidant power (FRAP) assays evaluating the chain-breaking antioxidant potential and the reducing power of the sample. Dietary TAC assessment by the Food Frequency Questionnaire was previously validated against TRAP, FRAP, and TEAC values estimated by a 3-day weighed food record and plasma TEAC and FRAP in a group of healthy Italian adults. ${ }^{20}$

\section{Postoperative Atrial Fibrillation and Secondary Outcomes Assessment}

The primary end point was the occurrence of POAF as collected from medical records. POAF was defined as an episode of AF that lasted more than 5 minutes or clinically recognized. The AF surveillance covered the period of stay in the intensive care unit (ICU) and the following week in a cardiac postsurgery unit. Sixty-three percent of POAFs occurred on the second and third days after the surgery.

As secondary end points, occurrence of other postoperative arrhythmias (ventricular tachycardia or fibrillation, other supraventricular tachycardia), extracorporeal circulation time (only for on-pump surgeries), total number of days in the ICU, and total in-hospital stay after the open heart surgery were collected from medical records.

\section{Covariates}

A trained research nurse measured blood pressure by an automatic device (OMRON HEM-705CP; Omron Healthcare Inc, Bannockburn, Ill) 3 times on the nondominant arm, and the average of the last 2 values was taken as the blood pressure value. Body weight and height measurements were made using standardized procedures. Body mass index was calculated as kilograms/meters squared. ${ }^{21}$

Subjects were classified as never smokers if they had never smoked cigarettes, former smokers if they had smoked cigarettes in the past but had 
TABLE 1. Dietary intake parameters of men and women undergoing cardiac surgery

\begin{tabular}{lcccc}
\hline \multicolumn{1}{c}{ Characteristics } & All $(\mathbf{N}=\mathbf{2 1 7})$ & Men $(\mathbf{N}=\mathbf{1 6 1})$ & Women $(\mathbf{N}=\mathbf{5 6})$ & \multicolumn{1}{c}{$\boldsymbol{P}$ value* } \\
\hline TEAC $($ mmol Trolox) & $5.35(3.7-7.6) \dagger$ & $6.2(4.4-8.5)$ & $3.6(2.5-4.2)$ & $<.0001$ \\
TRAP (mmol Trolox) & $7.45(5.2-10.5)$ & $8.8(6.2-12.2)$ & $5.2(3.3-6.6)$ & $<.0001$ \\
FRAP $\left(\mathrm{mmol} \mathrm{Fe}{ }^{2+}\right)$ & $15.6(11.1-22.6)$ & $18.0(13.4-24.5)$ & $10.7(7.5-13.2)$ \\
Total energy intake (kcal/d) & $1936.2(1533.5-2471.6)$ & $2169.3(1721.4-2604.5)$ & $1413.6(1152.7-1778.1)$ & $<.0001$ \\
\hline
\end{tabular}

FRAP, Ferric reducing antioxidant power; $T E A C$, Trolox equivalent antioxidant capacity; $T R A P$, radical-trapping antioxidant parameter. $* P$ value for the difference between men and women. $\dagger$ Median (interquartile range) (all such values).

stopped smoking since at least 1 year, and current smokers if they were currently smoking 1 or more cigarettes per day on a regular basis. Total physical activity (leisure and working time) was classified in metabolic equivalents per day. ${ }^{22}$

History of hypertension, diabetes, and hypercholesterolemia or previous cardiovascular events was collected by patient interview and by checking medical records. Preoperative and chronic pharmacologic therapies and surgery characteristics were collected from medical records.

\section{Study Population}

Until September 2012, 319 patients were invited to participate in the study among those hospitalized for a selective open heart surgery. Twenty-eight patients refused to participate (13\% were men; mean age $73.1 \pm 8.4$ years); the main motivations were preoperative anxiety $(\mathrm{N}=16)$, reduced degree of understanding $(\mathrm{N}=5)$, and unjustified refusal $(\mathrm{N}=5) ; 3$ patients were unable to sign the informed consent because of the severity of their clinical conditions.

TABLE 2. Baseline and operative characteristics of patients according to tertiles of Trolox equivalent antioxidant capacity intake

\begin{tabular}{|c|c|c|c|c|c|c|}
\hline Characteristics & All $(\mathbf{N}=\mathbf{2 1 7})$ & T1 $(N=71)$ & T2 $(N=73)$ & $\mathbf{T 3}(\mathrm{N}=73)$ & $P$ value & $P$ value \\
\hline Age, y & $68.4(62.7-73.8) \dagger$ & $69.1(61.7-73.8)$ & $70.0(64.0-74.3)$ & $66.0(60.8-71.5)$ & .19 & - \\
\hline Age $\geq 65$ y, N (\%) & $139(64.1)$ & $45(63.4)$ & $52(71.2)$ & $42(57.5)$ & .22 & - \\
\hline Gender, men, $\mathrm{N}(\%)$ & $161(74.2)$ & $53(74.7)$ & $54(74.0)$ & $54(74.0)$ & .99 & - \\
\hline Education, N (\%) & & & & & .10 & .16 \\
\hline Low & $154(71.0)$ & $52(73.2)$ & $58(79.4)$ & $44(60.3)$ & & \\
\hline Medium & $38(17.5)$ & $13(18.3)$ & $9(12.3)$ & 16 (21.9) & & \\
\hline High & $25(11.5)$ & $6(8.45)$ & $6(8.3)$ & $13(17.8)$ & & \\
\hline Smoking, N (\%) & & & & & .02 & .02 \\
\hline No & 91 (41.9) & $26(36.6)$ & $41(56.2)$ & 24 (32.9) & & \\
\hline Current & $29(13.4)$ & $7(9.9)$ & $8(11.0)$ & $14(19.2)$ & & \\
\hline Former & $97(44.7)$ & $38(53.5)$ & 24 (32.9) & 35 (47.9) & & \\
\hline BMI, $\mathrm{kg} / \mathrm{m}^{2}$ & $27.1(24.8-30.0)$ & $27.1(25.0-29.1)$ & $27.4(24.7-30.1)$ & $26.8(25.0-30.2)$ & .82 & .69 \\
\hline BMI, N (\%) & & & & & .48 & .59 \\
\hline Normal & $60(27.7)$ & $18(25.3)$ & $23(31.5)$ & $19(26.0)$ & & \\
\hline Overweight & $102(47.0)$ & $39(54.9)$ & $30(41.1)$ & $33(45.2)$ & & \\
\hline Obese & $55(25.3)$ & $14(19.72)$ & $20(27.4)$ & $21(28.8)$ & & \\
\hline Physical activity, N (\%) & & & & & .05 & .06 \\
\hline Low & $71(32.8)$ & $31(43.6)$ & $15(20.6)$ & $25(34.2)$ & & \\
\hline Medium & 73 (33.6) & $22(31.0)$ & $28(38.4)$ & $23(31.6)$ & & \\
\hline High & $73(33.6)$ & $18(25.3)$ & $30(41.1)$ & $25(34.2)$ & & \\
\hline Hypertension, N (\%) & $119(54.8)$ & $43(60.6)$ & $37(50.7)$ & $39(53.4)$ & .47 & .43 \\
\hline Diabetes, N (\%) & $70(32.3)$ & $27(38.0)$ & $18(24.7)$ & $25(34.2)$ & .21 & .19 \\
\hline Hypercholesterolemia, N (\%) & $56(25.8)$ & $22(31.0)$ & $15(20.6)$ & $19(26.0)$ & .36 & .40 \\
\hline History of arrhythmia, N (\%) & $74(34.1)$ & $23(32.4)$ & $25(34.2)$ & $26(35.6)$ & .92 & .80 \\
\hline History of AMI, N (\%) & $46(21.2)$ & $19(26.8)$ & $14(19.2)$ & $13(17.8)$ & .37 & .90 \\
\hline Use of beta-blockers, $\mathrm{N}(\%)$ & $142(65.4)$ & $48(67.6)$ & 45 (61.6) & $49(67.1)$ & .70 & .69 \\
\hline Use of ACE inhibitors, $\mathrm{N}(\%)$ & $135(62.2)$ & $47(66.2)$ & $44(60.3)$ & $44(60.3)$ & .70 & .43 \\
\hline Use of statins, $N(\%)$ & $122(56.2)$ & $43(60.6)$ & $37(50.7)$ & $42(57.5)$ & .47 & .31 \\
\hline Use of hypoglycemic drugs, $\mathrm{N}(\%)$ & $54(24.9)$ & $22(31.0)$ & $11(15.1)$ & $21(28.8)$ & .06 & .05 \\
\hline Diet for chronic disease, $\mathrm{N}(\%)$ & $28(12.9)$ & $15(21.1)$ & $4(5.5)$ & $9(12.3)$ & .02 & .06 \\
\hline Type surgery, N (\%) & & & & & .50 & .25 \\
\hline CABG only & $110(50.7)$ & $37(52.1)$ & $34(46.6)$ & $39(53.4)$ & & \\
\hline VR only & $54(24.9)$ & $15(21.1)$ & $18(24.7)$ & $21(28.8)$ & & \\
\hline Combined CABG and VR & $29(13.4)$ & $12(16.9)$ & $9(12.3)$ & $8(11.0)$ & & \\
\hline Other open heart surgery & $24(11.0)$ & $7(9.9)$ & $12(16.4)$ & $5(6.9)$ & & \\
\hline Off-pump surgery, $\mathrm{N}(\%)$ & $12(5.5)$ & $7(9.9)$ & $1(1.4)$ & $4(5.5)$ & .07 & .24 \\
\hline
\end{tabular}

$A C E$, Angiotensin-converting enzyme; $A M I$, acute myocardial infarction; $B M I$, body mass index; $C A B G$, coronary artery bypass grafting; $T 1$, first tertile; $T 2$, second tertile; $T 3$, third tertile; $V R$, valve replacement or repair. *Multivariable 1 adjusted for age, gender, and caloric intake. $\dagger$ Median (interquartile range) (all such values). 
TABLE 3. Odds ratios of developing postoperative atrial fibrillation after a cardiac surgery, according to Trolox equivalent antioxidant capacity tertiles

\begin{tabular}{|c|c|c|c|c|c|c|}
\hline Type of cardiac surgeries & POAF $(\%)$ & Crude OR $(95 \%$ CI $)$ & $P$ value & $P$ for trend & $\begin{array}{c}\text { Multivariable } 1 \text { OR } \\
(95 \% \text { CI })\end{array}$ & $P$ value \\
\hline \multicolumn{7}{|l|}{ All open surgeries, $\mathrm{N}=217$} \\
\hline $\mathrm{T} 1(\mathrm{~N}=71)$ & $31(43.7)$ & 1 & & .044 & 1 & \\
\hline $\mathrm{T} 2(\mathrm{~N}=73)$ & $33(43.8)$ & $1.06(0.55-2.05)$ & .85 & & $1.10(0.54-2.2)$ & .79 \\
\hline $\mathrm{T} 3(\mathrm{~N}=73)$ & $19(26.0)$ & $0.45(0.22-0.92)$ & .027 & & $0.51(0.22-1.15)$ & .10 \\
\hline \multicolumn{7}{|l|}{ a) CABG/VR, N = 193} \\
\hline $\mathrm{T} 1(\mathrm{~N}=64)$ & $29(45.3)$ & 1 & & .039 & 1 & \\
\hline $\mathrm{T} 2(\mathrm{~N}=61)$ & $29(47.5)$ & $1.09(0.54-2.21)$ & .80 & & $1.03(0.48-2.19)$ & .94 \\
\hline $\mathrm{T} 3(\mathrm{~N}=68)$ & $18(26.5)$ & $0.43(0.21-0.90)$ & .025 & & $0.41(0.17-0.97)$ & .043 \\
\hline \multicolumn{7}{|l|}{ b) Isolated CABG, $N=110$} \\
\hline $\mathrm{T} 1(\mathrm{~N}=37)$ & $13(35.1)$ & 1 & & .25 & 1 & \\
\hline $\mathrm{T} 2(\mathrm{~N}=34)$ & $14(40.0)$ & $1.29(0.49-3.38)$ & 60 & & $1.14(0.39-3.28)$ & .81 \\
\hline $\mathrm{T} 3(\mathrm{~N}=39)$ & $8(20.5)$ & $0.48(0.17-1.33)$ & .16 & & $0.39(0.10-1.44)$ & .16 \\
\hline
\end{tabular}

Multivariable 1 adjusted for age, gender, caloric intake; multivariable 2 adjusted for age, gender, caloric intake, use of hypoglycemic drug, education, previous diagnosis of AF, and physical activity. $C A B G$, Coronary artery bypass grafting; $P O A F$, postoperative atrial fibrillation; $T 1$, first tertile; $T 2$, second tertile; $T 3$, third tertile; $T 12$, sum first and second tertiles; $V R$, valve replacement or repair; $C I$, confidence interval; $O R$, odds ratio.

A total of 31 patients were excluded because they did not undergo the planned open heart surgery, and 32 patients were excluded because of noncompliance with the inclusion criteria or incomplete data collection, 8 because of reporting an extreme energy intake (lower or upper percentiles of the energy intake distribution). Finally, 217 patients were analyzed.

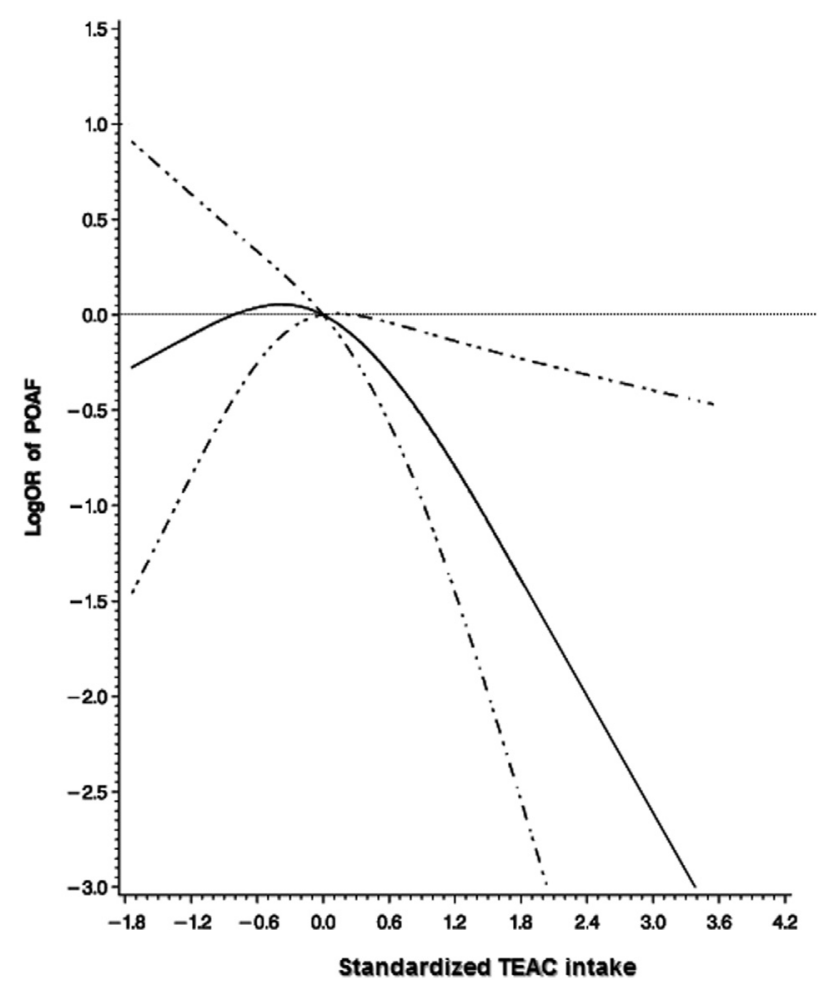

FIGURE 1. Relationship between standardized TEAC and risk of POAF fitted with natural cubic splines. The reference line $(\log \mathrm{OR}, 0)$ goes through the zero value of the standardized TEAC distribution; the solid line is for $\log$ ORs; the dashed lines are for $95 \% \mathrm{CI}$ boundaries. POAF, Postoperative atrial fibrillation; TEAC, Trolox equivalent antioxidant capacity; $O R$, odds ratio.

\section{Statistical Analysis}

Dietary TAC, assessed as TEAC, TRAP, or FRAP, was categorized into tertiles on the basis of sex-specific distribution or reported as median (interquartile range). The association between tertile of dietary TAC and POAF was assessed by logistic multivariable analysis in the whole sample and separately for subgroups of cardiac surgeries. The basic model was adjusted for all covariates associated with both POAF and TAC intake, with a significance level of at least $P=.2$. The final multivariable models included age, gender, caloric intake, use of hypoglycemic drugs, physical activity, education, and previous diagnosis of AF. The statistical significance of potential interaction between dietary TAC in tertiles and specific covariates (ie, use of beta-blockers or angiotensin-converting enzyme inhibitors) was tested using the Wald test for a multiplicative interaction term.

FRAP, TEAC, and TRAP are 3 indicators of dietary TAC, strongly correlated with each other $(r=0.98 ; P<.0001)$. To avoid redundancy in the presentation of data, we used the indicator that fit the data the best in our analyses. For the goodness of fit test, we used the Akaike information criterion $^{12,23}$; the lower the Akaike information criterion, the better the goodness of fit. Because TEAC showed the lowest Akaike information criterion compared with FRAP and TRAP, it was selected as the better indicator of dietary TAC.

Natural cubic splines were fitted to logistic regression models to examine the relationship between TAC (TEAC variable as continuous variable) and risk of POAF. In this analysis, TEAC intake was standardized to mean equal to zero and standard deviation equal to 1 separately for men and women. A SAS (SAS Institute Inc, Cary, NC) macro named "lgtphcurv9" "24 was used, which implements natural cubic spline methodology to fit potential nonlinear dose-response curves in logistic regression models. Likelihood ratio tests were performed to test nonlinear and linear relations. ${ }^{25}$ The zero value of the standardized TAC values was used as reference.

Sensitivity analyses were performed by subgroups of cardiac surgeries: CABG and/or VR $(\mathrm{N}=193)$ and isolated CABG $(\mathrm{N}=110)$. The contribution of single food groups to total dietary TAC was assessed by stepwise multiple regression analysis.

The sample size of 217 patients undergoing cardiac surgery (fixing $\alpha=0.05$ and $\beta=0.2$, and considering the incidence of $\mathrm{POAF}=30 \%)^{3,4}$ was able to detect an odds ratio (OR) of 0.45 or less. ${ }^{26}$ The analyses were performed by SAS 9.1 .3 for Windows (SAS Institute Inc). 
TABLE 3. Continued

\begin{tabular}{|c|c|c|c|c|c|c|c|}
\hline$P$ for trend & $\begin{array}{c}\text { Multivariable } 2 \text { OR } \\
(95 \% \text { CI })\end{array}$ & $P$ value & $P$ for trend & Type of cardiac surgeries & POAF $(\%)$ & $\begin{array}{c}\text { Multivariable } 2 \text { OR } \\
(95 \% \text { CI })\end{array}$ & $P$ value \\
\hline & & & & All open surgeries, $\mathrm{N}=217$ & & & \\
\hline \multirow[t]{4}{*}{.12} & 1 & & .11 & & & & \\
\hline & $1.04(0.49-2.21)$ & .91 & & $\mathrm{~T} 12(\mathrm{~N}=144)$ & $64(44.4)$ & 1 & \\
\hline & $0.47(0.19-1.13)$ & .09 & & $\mathrm{~T} 3(\mathrm{~N}=73)$ & $19(26.0)$ & $0.46(0.22-0.95)$ & .048 \\
\hline & & & & a) $\mathrm{CABG} / \mathrm{VR}, \mathrm{N}=193$ & & & \\
\hline \multirow[t]{4}{*}{.051} & 1 & & .047 & & & & \\
\hline & $1.01(0.45-2.24)$ & .98 & & $\mathrm{~T} 12(\mathrm{~N}=125)$ & $58(46.4)$ & 1 & \\
\hline & $0.38(0.15-0.94)$ & .037 & & $\mathrm{~T} 3(\mathrm{~N}=68)$ & $18(26.5)$ & $0.37(0.17-0.81)$ & .013 \\
\hline & & & & b) Isolated CABG, $N=110$ & & & \\
\hline \multirow[t]{3}{*}{.21} & 1 & & .18 & & & & \\
\hline & $1.30(0.41-4.15)$ & .66 & & $\mathrm{~T} 12(\mathrm{~N}=71)$ & $27(38.0)$ & 1 & \\
\hline & $0.35(0.08-1.44)$ & .15 & & $\mathrm{~T} 3(\mathrm{~N}=39)$ & $8(20.5)$ & $0.29(0.09-0.94)$ & .04 \\
\hline
\end{tabular}

\section{RESULTS}

TAC intake in male and female patients is shown in Table 1. Compared with men, women showed lower levels of dietary TEAC, TRAP, FRAP, and total energy intake.

Table 2 lists the main characteristics of the recruited patients in the whole sample and according to tertiles of TEAC intake. Some $74 \%$ of patients were men; the median age was 68.4 years, and $64 \%$ of patients were aged more than 65 years. There were no differences among tertiles of TEAC intake in the main characteristics of the population, except for smoking habits and physical activity: The prevalence of smokers was higher in the third tertile of TEAC distribution, whereas subjects with a low physical activity were more prevalent in the first tertile.

In the whole sample, the incidence of total arrhythmias and POAF was $42.4 \%$ and $38.2 \%$, respectively. In multivariable analysis, after adjustment for age, sex, use of hypoglycemic drugs, physical activity, education, previous diagnosis of AF, and total energy intake, patients in the highest tertile of TEAC (T3: $\mathrm{N}=73$ ) had a lower risk of POAF than patients in the 2 lowest tertiles (T12: $\mathrm{N}=144)(\mathrm{OR}, 0.46 ; 95 \%$ confidence interval $[\mathrm{CI}], 0.22-0.95 ; P=.048$ ) (Table 3 ).

In subgroup analyses, considering (1) CABG and/or VR and combined surgeries $(\mathrm{N}=193)$ and $(2)$ isolated CABG $(\mathrm{N}=110)$, the protective effect of a diet rich in antioxidants on POAF was confirmed in both groups: $\mathrm{OR}_{\mathrm{a}}, 0.37$ (95\% CI, 0.17-0.81; $P=.013)$ and $\mathrm{OR}_{\mathrm{b}}, 0.29$ (95\% CI, 0.09$0.94, P=.04)$, respectively. Excluding "off-pump" cardiac surgeries that were not performed in extracorporeal circulation $(\mathrm{N}=12)$ did not change the results $(\mathrm{OR}, 0.45 ; 95 \% \mathrm{CI}$, $0.21-0.95 ; P=.036$ ). When using the Wald test, no statistical significance of potential interaction was found between dietary TAC and use of beta-blockers or angiotensinconverting enzyme inhibitors $(P=.99$ and $P=.70$, respectively).
A restricted cubic spline transformation confirmed the nonlinear relationship between TAC and risk of POAF $(P=.023)$ (Figure 1). Values of TAC below the sexspecific mean value (corresponding to zero value of the standardized TAC used in Figure 1 as reference value) were not associated with the risk of POAF; higher values of TAC were associated with increasing protection against POAF (Figure 1).

The incidence of total postoperative arrhythmias was not significantly lower in the third tertile of TAC after adjusted analysis $(32.9 \%$ vs $47.3 \%)$ (Table 4$)$. When considering other secondary outcomes, there were no significant differences in the extracorporeal circulation time (only on-pump surgeries $\mathrm{N}=205$ ), total number of days in the ICU, or total in-hospital stay after open heart surgery among the tertile groups; however, the latter 2 showed a trend to be reduced in patients in the highest tertile of TAC intake (Table 4).

To explore the relative contribution of food groups to total dietary TAC, we performed stepwise multiple regression analysis with TAC intake as a dependent variable and intake of cereals, vegetables, legumes, oils and nuts, fruit and fruit juices, coffee, tea, chocolate, wine, and other alcoholic beverages in grams per day as independent variables, while controlling for age and gender.

As shown in Table 5, the dietary intake of all mentioned food groups explained more than $85 \%$ of total dietary TAC variance. Wine $(46.5 \%)$ and coffee $(29.7 \%)$ represented the main sources of dietary TAC (TEAC) in our population.

\section{DISCUSSION}

In this prospective analysis, we have shown, possibly for the first time, that a diet rich in antioxidants, reportedly consumed during the last year, is associated with a reduced risk of POAF in patients undergoing cardiac surgery (mainly CABG and VR). Previous epidemiologic studies 
TABLE 4. Secondary outcomes according to Trolox equivalent antioxidant capacity tertiles

\begin{tabular}{|c|c|c|c|c|c|}
\hline Secondary outcomes & All $(N=217)$ & $\mathrm{T12}(\mathrm{N}=144)$ & $\mathbf{T 3}(\mathrm{N}=\mathbf{7 3})$ & $P$ value & $P$ value* \\
\hline Total arrhythmias, $\mathrm{N}(\%)$ & $92(42.4)$ & $68(47.3)$ & $24(32.9)$ & .04 & .23 \\
\hline Ventricular tachycardia or fibrillation, $\mathrm{N}(\%)$ & $5(2.3)$ & $2(1.4)$ & $3(4.1)$ & .33 & .15 \\
\hline Other supraventricular tachycardia, N (\%) & $4(1.8)$ & $3(2.1)$ & $1(1.4)$ & .99 & .65 \\
\hline Total ICU stay (d), mean (SD) & $2.6(3.1)$ & $2.8(3.7)$ & $2.3(1.0)$ & .31 & .41 \\
\hline Total in-hospital stay (d), mean (SD) & $8.6(11.2)$ & $9.1(13.3)$ & $7.7(4.7)$ & .34 & .50 \\
\hline Patients with any POAF & $\mathrm{N}=83$ & $\mathrm{~N}=64$ & $\mathrm{~N}=19$ & & \\
\hline Total ICU stay with any POAF (d), mean (SD) & $3.1(4.7)$ & $3.3(5.3)$ & $2.5(1.4)$ & .38 & .78 \\
\hline Total in-hospital stay with any POAF (d), mean (SD) & $10.6(17.1)$ & $11.4(19.4)$ & $8.0(3.9)$ & .51 & .83 \\
\hline No. of on-pump surgeries & $\mathrm{N}=205$ & $\mathrm{~N}=136$ & $\mathrm{~N}=69$ & & \\
\hline \multicolumn{6}{|l|}{ ECC time (min) } \\
\hline Mean (SD) & $84.3(28.59)$ & $84.3(30.7)$ & $83.7(25.1)$ & .83 & .29 \\
\hline Median (IQR) & 78 (65-99) & $78(64.5-101)$ & $80(65-95)$ & & \\
\hline
\end{tabular}

$E C C$, Extracorporeal circulation; $I C U$, intensive care unit; $I Q R$, interquartile range; $P O A F$, postoperative atrial fibrillation; $S D$, standard deviation; $T 12$, sum first and second tertiles; $T 3$, third tertile. *Multivariable 1 adjusted for age, gender, and caloric intake.

have shown that dietary TAC is inversely related to metabolic syndrome, total mortality, ${ }^{12}$ stroke, ${ }^{13}$ and myocardial infarction. $^{14}$

Foods rich in antioxidants are typical of a Mediterraneanstyle diet. The beneficial effects of Mediterranean diet are widely recognized, and several observational studies suggested that people with high intakes of fruits, vegetables, and olive oil, which are rich in antioxidants, vitamins $\mathrm{C}$ and $\mathrm{E}$, beta-carotene, selenium, alpha-tocopherol, and flavonoids, showed a lower risk of chronic cardiovascular and cerebrovascular disease. ${ }^{27-29}$ In a recent case-control study, low adherence to a Mediterranean diet was positively associated with AF and a lower probability of a spontaneous conversion of $\mathrm{AF}^{30}$

A strong increase in inflammatory markers, such as interleukin-1, C-reactive protein, fibrinogen, and plasminogen activator inhibitor-1, was shown in the first 48 hours after an open heart surgery and was related to the incidence of POAF. On the other hand, a high dietary TAC intake was associated with decreased levels of C-reactive protein. ${ }^{10,31}$ The possible mechanisms implicated in risk reduction of POAF by dietary antioxidants may be related to protection against increased oxidative stress and inflammation, which are involved in causing POAF. During open heart surgery, a strong and persistent inflammatory condition is likely to occur, due to both the nature of the disease and the influence of the operation techniques. In our study, the inverse association between dietary antioxidants and occurrence of POAF was similar in combined $\mathrm{CABG}$ and $\mathrm{VR}$ or isolated $\mathrm{CABG}$, suggesting that this was related to a condition induced by the open heart surgery rather than to the underlying disease.

Supplementation with antioxidant vitamins or antioxidant reinforcement of omega-3 polyunsaturated fatty acids therapy seems to reduce the occurrence of POAF after cardiac surgery, although contrasting results have been reported. 9,32 Another possible mechanism of decreased AF by dietary antioxidants is the increase in omega- 3 content of plasma and red cell membranes. Indeed, the intake of dietary polyphenols was associated with high plasma and red blood cell concentrations of marine n-3 fatty acids. ${ }^{33}$

The food items that contributed the most to dietary TAC variance in this population were wine, coffee, and fruit, which altogether explained more than $85 \%$ of the total antioxidant intake. Red wine was the major contributor to dietary TAC (Table 5) because it contains a wide variety of

TABLE 5. Contribution of selected food groups to dietary total antioxidant capacity*

\begin{tabular}{|c|c|c|c|}
\hline Characteristics & TEAC (mmol Trolox) $\%$ & TRAP (mmol Trolox) \% & FRAP $\left(\mathrm{mmol} \mathrm{Fe}^{2+}\right) \%$ \\
\hline Wine & 46.47 & 37.67 & 41.25 \\
\hline Coffee & 29.67 & 45.90 & 36.61 \\
\hline Fruit and fruit juices & 8.10 & 3.90 & 7.95 \\
\hline Chocolate & 2.52 & 1.14 & 1.27 \\
\hline Cereals & 1.21 & 0.88 & 1.19 \\
\hline Other alcoholic beverages & 0.07 & 0.33 & 1.68 \\
\hline Tea & 0.05 & 0.40 & 0.51 \\
\hline Vegetables, oils, nuts, legumes & $<0.1$ & 0.18 & $<0.1$ \\
\hline
\end{tabular}

$\overline{F R A P \text {, Ferric reducing antioxidant power; TEAC, Trolox equivalent antioxidant capacity; TRAP, radical-trapping antioxidant parameter. *Percentages represent the partial } R^{2}}$ obtained from a linear regression model (controlled for age and sex) to explain the contribution of a variable xj(food) to the explanation of the variation of a dependent variable y (dietary TEAC, TRAP, and FRAP, respectively). 
polyphenols with antioxidant and anti-infiammatory properties. ${ }^{34,35}$ It is somewhat controversial whether caffeine consumption is associated with an increased risk of developing $\mathrm{AF}^{36,37}$ However, a recent meta-analysis has shown that exposure to low doses of caffeine may offer a small protective effect against $\mathrm{AF}^{37}$

Current guidelines recommend beta-blockers as the firstline preventive treatment of AF after cardiac surgery; if beta-blocker therapy is contraindicated, then amiodarone is recommended. ${ }^{38}$ The 2 drugs show a similar limited effect on the occurrence of AF and length of hospital stay after surgery so that newer upstream therapies (drugs or nutraceuticals) have been investigated ( $\mathrm{n}-3$ polyunsaturated fatty acids, colchicine, and statin therapy), although with contrasting results. ${ }^{8,9}$

The relationship we observed was nonlinear, because the lower intake of TAC (below the mean value of sex-specific standardized TEAC distribution) was not associated with POAF, but a higher consumption could reduce the risk of POAF. Recommendations on dietary habits in patients with coronary artery disease at high risk to undergo a cardiac surgery are uncommon. In view of our results, dietary habits may be of particular relevance in preventing the arrhythmia, in addition to the traditional antiarrhythmic therapy.

\section{Study Limitations}

A major limitation of the present study is the relatively small number of recruited patients; however, this did not prevent statistically significant differences to be consistently observed. Moreover, the incidence of POAF events was comparable to those reported by other studies in this field. ${ }^{9}$

This study was not an intervention study, and we cannot completely rule out the effect of unmeasured (or unknown) confounding factors. For example, a genetic predisposition for electrophysiologic and conduction abnormalities could be associated with POAF occurrence; however, it is hard to suppose that such a genetic background is associated with TAC intake, and thus we can exclude it as a possible confounding variable. Similar considerations also can apply to differences in pre- and posthemodynamic and cardiac procedures for myocardial protection.

Dietary TAC was measured using food frequency questionnaires; therefore, some measurement error in dietary TAC is unavoidable. Moreover, TAC is a parameter measured in vitro and does not necessarily relate to the in vivo antioxidant potential, because some dietary antioxidants may be poorly absorbed after ingestion. On the other hand, in comparison with observational and prospective studies with longer follow-ups, ${ }^{12-14}$ TAC intake was measured during the preoperative hospital stay; therefore, the measurement was precise and could not be affected by lifestyle changes due to the disease.

\section{CONCLUSIONS}

This study suggests that a diet rich in antioxidants, reflecting at least 1 year of healthy dietary habits, may be associated with a reduction in the risk of $\mathrm{AF}$ after cardiac surgery. The major contributors to dietary TAC were wine, coffee, and fruits. Additional and larger studies of the association between dietary TAC and POAF are needed, as well as studies evaluating whether a short-term preoperative supplementation of an antioxidant-rich food might offer significant protection for POAF occurrence after cardiac surgery.

\section{References}

1. Ahlsson A, Bodin L, Fengsrud E, Englund A. Patients with postoperative atrial fibrillation have a doubled cardiovascular mortality. Scand Cardiovasc J. 2009; 43:330-6.

2. Lahtinen J, Biancari F, Salmela E, Mosorin M, Satta J, Rainio P, et al. Postoperative atrial fibrillation is a major cause of stroke after on-pump coronary artery bypass surgery. Ann Thorac Surg. 2004;77:1241-4.

3. Maisel WH, Rawn JD, Stevenson WG. Atrial fibrillation after cardiac surgery. Ann Intern Med. 2001;135:1061-73.

4. Echahidi N, Pibarot P, O’Hara G, Mathieu P. Mechanisms, prevention, and treatment of atrial fibrillation after cardiac surgery. J Am Coll Cardiol. 2008;51: 793-801.

5. Banach M, Rysz J, Drozdz JA, Okonski P, Misztal M, Barylski M, et al. Risk factors of atrial fibrillation following coronary artery bypass grafting: a preliminary report. Circ J. 2006;70:438-41.

6. Gaudino M, Andreotti F, Zamparelli R, Di Castelnuovo A, Nasso G, Burzotta F, et al. The $-174 \mathrm{G} / \mathrm{C}$ interleukin-6 polymorphism influences postoperative interleukin-6 levels and postoperative atrial fibrillation: is atrial fibrillation an inflammatory complication? Circulation. 2003;108(Suppl 1):II195-9.

7. Elahi MM, Flatman S, Matata BM. Tracing the origins of postoperative atrial fibrillation: the concept of oxidative stress-mediated myocardial injury phenomenon. Eur J Cardiovasc Prev Rehabil. 2008;15:735-41.

8. Savelieva I, Kakouros N, Kourliouros A, Camm AJ. Upstream therapies for management of atrial fibrillation: review of clinical evidence and implications for European Society of Cardiology guidelines, part I: primary prevention. Europace. 2011;13:308-28

9. Costanzo S, di Niro V, Di Castelnuovo A, Gianfagna F, Donati MB, de Gaetano G, et al. Prevention of postoperative atrial fibrillation in open heart surgery patients by preoperative supplementation of $n-3$ polyunsaturated fatty acids: an updated meta-analysis. J Thorac Cardiovasc Surg. 2013;146:906-11.

10. Brighenti F, Valtueña S, Pellegrini N, Ardigò D, Del Rio D, Salvatore S, et al. Total antioxidant capacity of the diet is inversely and independently related to plasma concentration of high-sensitivity C-reactive protein in adult Italian subjects. Br J Nutr. 2005;93:619-25.

11. Bahadoran Z, Golzarand M, Mirmiran P, Shiva N, Azizi F. Dietary total antioxidant capacity and the occurrence of metabolic syndrome and its components af ter a 3-year follow-up in adults: Tehran Lipid and Glucose Study. Nutr Metab (Lond). 2012;9:70.

12. Agudo A, Cabrera L, Amiano P, Ardanaz E, Barricarte A, Berenguer T, et al. Fruit and vegetable intakes, dietary antioxidant nutrients, and total mortality in Spanish adults: findings from the Spanish cohort of the European Prospective Investigation into Cancer and Nutrition (EPIC-Spain). Am J Clin Nutr. 2007;85: 1634-42.

13. Del Rio D, Agnoli C, Pellegrini N, Krogh V, Brighenti F, Mazzeo T, et al. Total antioxidant capacity of the diet is associated with lower risk of ischemic stroke in a large Italian cohort. J Nutr. 2011;141:118-23.

14. Rautiainen S, Levitan EB, Orsini N, ̊kkesson A, Morgenstern R, Mittleman MA et al. Total antioxidant capacity from diet and risk of myocardial infarction: a prospective cohort of women. Am J Med. 2012;125:974-80.

15. Pala V, Sieri S, Palli D, Salvini S, Berrino F, Bellegotti M, et al. Diet in the Italian EPIC cohorts: presentation of data and methodological issues. Tumori. 2003;89: 594-607.

16. Salvini S, Parpinel M, Gnagnarella P, Maisonneuve P, Turrini A. Banca Dati di Composizione degli Alimenti per Studi Epidemiologici in Italia. Milan, Italy: Istituto Europeo di Oncologia; 1998. 
17. Pellegrini N, Serafini M, Salvatore S, Del Rio D, Bianchi M, Brighenti F. Total antioxidant capacity of spices, dried fruits, nuts, pulses, cereals and sweets consumed in Italy assessed by three different in vitro assays. Mol Nutr Food Res. 2006;50:1030-8.

18. di Giuseppe R, Arcari A, Serafini M, Di Castelnuovo A, Zito F, De Curtis A, et al. Total dietary antioxidant capacity and lung function in an Italian population: a favorable role in premenopausal/never smoker women. Eur J Clin Nutr. 2012;66:61-8.

19. Detopoulou P, Panagiotakos DB, Chrysohoou C, Fragopoulou E, Nomikos T, Antonopoulou S, et al. Dietary antioxidant capacity and concentration of adiponectin in apparently healthy adults: the ATTICA study. Eur J Clin Nutr. 2010;64: $161-8$.

20. Pellegrini N, Salvatore S, Valtueña S, Bedogni G, Porrini M, Pala V, et al. Development and validation of a food frequency questionnaire for the assessment of dietary total antioxidant capacity. J Nutr. 2007;137:93-8.

21. Iacoviello L, Bonanni A, Costanzo S, De Curtis A, Di Castelnuovo A, Olivieri M, et al. The Moli-Sani Project, a randomized, prospective cohort study in the Molise region in Italy; design, rationale and objectives. Ital J Public Health. 2007;4 $110-8$

22. Centritto F, Iacoviello L, di Giuseppe R, De Curtis A, Costanzo S, Zito F, et al. Dietary patterns, cardiovascular risk factors and C-reactive protein in a healthy Italian population. Nutr Metab Cardiovasc Dis. 2009;19:697-706.

23. Akaike H. Information theory and an extension of the maximum likelihood principle. In: Petrov BN, Csaki F, eds. Proceedings of the Second International Symposium on Information Theory. Budapest: Akademiai Kiado; 1973:267-81.

24. Li R, Hertzmark E, Louie M, Chen L, Spiegelman D. The SAS LGTPHCURV9 Macro. Available at: http://www.hsph.harvard.edu/spiegelman/lgtphcurv9/ lgtphcurv9.pdf. Accessed September 6, 2014

25. Durrleman S, Simon R. Flexible regression models with cubic splines. Stat Med. 1989;8:551-61.

26. Dupont WD, Plummer W. Power and sample size calculations for studies involving linear regression. Control Clin Trials. 1998;19:589-601.

27. Yusuf S, Hawken S, Ounpuu S, Dans T, Avezum A, Lanas F, et al. Effect of potentially modifiable risk factors associated with myocardial infarction in 52 countries (the INTERHEART study): case-control study. Lancet. 2004;364:937-52.
28. Bonaccio M, Iacoviello L, de Gaetano G, on behalf of the Moli-Sani Investigators. The Mediterranean diet: the reasons for a success. Thromb Res. 2012;129: 401-4.

29. Sofi F, Cesari F, Abbate R, Gensini GF, Casini A. Adherence to Mediterranean diet and health status: meta-analysis. BMJ. 2008;337:a1344.

30. Mattioli AV, Miloro C, Pennella S, Pedrazzi P, Farinetti A. Adherence to Mediterranean diet and intake of antioxidants influence spontaneous conversion of atrial fibrillation. Nutr Metab Cardiovasc Dis. 2013;23:115-21.

31. Valtueña S, Pellegrini N, Franzini L, Bianchi MA, Ardigò D, Del Rio D, et al. Food selection based on total antioxidant capacity can modify antioxidant intake, systemic inflammation, and liver function without altering markers of oxidative stress. Am J Clin Nutr. 2008;87:1290-7.

32. Rodrigo R, Korantzopoulos P, Cereceda M, Asenjo R, Zamorano J, Villalabeitia E, et al. A randomized controlled trial to prevent post-operative atrial fibrillation by antioxidant reinforcement. J Am Coll Cardiol. 2013;62: 1457-65.

33. di Giuseppe R, de Lorgeril M, Salen P, Laporte F, Di Castelnuovo A, Krogh V, et al. Alcohol consumption and n-3 polyunsaturated fatty acids in healthy men and women from 3 European populations. Am J Clin Nutr. 2009;89:354-62.

34. Noguer MA, Cerezo AB, Donoso Navarro E, Garcia-Parrilla MC. Intake of alcohol-free red wine modulates antioxidant enzyme activities in a human intervention study. Pharmacol Res. 2012;65:609-14.

35. Costanzo S, Di Castelnuovo A, Donati MB, Iacoviello L, de Gaetano G. Wine, beer or spirit drinking in relation to fatal and non-fatal cardiovascular events: a meta-analysis. Eur J Epidemiol. 2011;26:833-5.

36. Di Castelnuovo A, di Giuseppe R, Iacoviello L, de Gaetano G. Consumption of cocoa, tea and coffee and risk of cardiovascular disease. Eur J Intern Med. 2012; 23:15-25.

37. Caldeira D, Martins C, Alves LB, Pereira H, Ferreira JJ, Costa J. Caffeine does not increase the risk of atrial fibrillation: a systematic review and meta-analysis of observational studies. Heart. 2013;99:1383-9.

38. Mitchell LB, CCS Atrial Fibrillation Guidelines Committee. Canadian Cardiovascular Society atrial fibrillation guidelines 2010: prevention and treatment of atrial fibrillation following cardiac surgery. Can J Cardiol. 2011;27:91-7.

\section{EDITORIAL COMMENTARY}

\section{Mediterranean-style diet to prevent postoperative atrial fibrillation: Role of antioxidants?}

Jun Feng, MD, PhD

See related article on pages $1175-82$.

From the Division of Cardiothoracic Surgery, Department of Surgery, Cardiovascular Research Center, Rhode Island Hospital, Alpert Medical School of Brown University, Providence, RI.

Disclosures: Author has nothing to disclose with regard to commercial support.

Received for publication Nov 21, 2014; accepted for publication Nov 22, 2014; available ahead of print Dec 19, 2014

Address for reprints: Jun Feng, MD, PhD, Division of Cardiothoracic Surgery, Cardiovascular Research Center, 1 Hoppin St, Coro West, Room 5.235, Providence, RI 02903 (E-mail: jfeng @ lifespan.org).

J Thorac Cardiovasc Surg 2015;149:1182-4

$0022-5223 / \$ 36.00$

Copyright $(\odot 2015$ by The American Association for Thoracic Surgery

http://dx.doi.org/10.1016/j.jtcvs.2014.11.063
During the past several decades, numerous epidemiologic studies and clinical trials have outlined a clear link between diet and cardiovascular events. Nowadays, physicians always tell their patients that "you are what you eat." A Mediterranean type of diet was first reported to be a healthy diet by Keys and colleagues. ${ }^{1}$ Today, the Mediterranean diet has been globally recognized as one of the healthiest diets in terms of its prevention of cardiovascular disease and metabolic syndrome. ${ }^{2,3}$ People living in the Mediterranean area choose olive oil as the main source of dietary fat, with moderate consumption of ethanol, low consumption of meat and meat products, and high consumption of vegetables, fish, fruits, nuts, whole grain, and legumes. Research has shown that the major components of the Mediterranean diet have potential protective properties, such as antioxidation and antiinflammation. ${ }^{4,5}$ 


\section{APPENDIX E1. OBSERVATIONAL POLYPHEMUS PROJECT INVESTIGATORS}

Principal Investigators: Licia Iacoviello, MD, PhD, Simona Costanzo, MSc, PhD

IRCCS Neuromed, Pozzilli, Italy: Marialaura Bonaccio, Americo Bonanni, Chiara Cerletti, Simona Costanzo, Amalia De Curtis, Giovanni de Gaetano, Augusto Di Castelnuovo, Maria Benedetta Donati, Licia Iacoviello, Mariarosaria Persichillo

Fondazione di Ricerca e Cura "Giovanni Paolo II," Campobasso, Italy: Cynthia Barilaro, Eugenio Caradonna, Eugenio Calvo, Vincenzo Cianci, Carlo Maria De Filippo, Nicola Testa, Bruno Zappacosta
Recruitment staff: Daniele Bredice (Campobasso), Francesca D'Aversa (Campobasso), Veronica Di Niro (Campobasso), Stefano Mercurio (Campobasso), Mariarosaria Morena (Campobasso), Teresa Panzera (Campobasso), Maria Zeoli (Campobasso)

Laboratory staff: Daniela Cugino (Campobasso), Antonella Cutrone (Campobasso), Marco D'Imperio (Campobasso), Iolanda Santimone (Campobasso)

Informatics: Marco Olivieri (Epicomed Research Srl, Campobasso, Italy) 